\title{
The cometary impactor flux at the Earth
}

\author{
Paul R. Weissman $\dagger$ \\ Science Division, Jet Propulsion Laboratory, Pasadena, CA 91109 USA \\ email: paul.r.weissman@jpl.nasa.gov
}

\begin{abstract}
Comets account for a small but very significant fraction of impactors on the Earth. Although the total number of Earth-crossing comets is modest as compared with asteroids, the more eccentric and inclined orbits of the comets result in much higher encounter velocities with the planet. Additionally, some Earth-crossing comets are significantly larger than any current near-Earth asteroids (NEAs); comets 1P/Halley and C/1995 O1 Hale-Bopp are good examples of this. Thus, the most energetic impacts on the Earth likely result from comets and not NEAs. The mean impact probability for long-period comets is $2.4 \times 10^{-9}$ per comet per perihelion passage, assuming the perihelion distribution of Everhart (1967), with a most probable encounter velocity of $53.5 \mathrm{~km} \mathrm{sec}^{-1}$. There are 21 known Earth-crossing Jupiter-family comets with a mean impact probability of $1.6 \times 10^{-9}$ per comet per year and a most probable encounter velocity of $17.0 \mathrm{~km} \mathrm{sec}^{-1}$. For the 16 known Earth-crossing Halley-type comets the mean impact probability is $1.2 \times 10^{-10}$ per year with a most probable encounter velocity of $51.3 \mathrm{~km} \mathrm{sec}^{-1}$. The poor knowledge of the size distribution of cometary nuclei makes it difficult to estimate actual impact energies at this time, though that situation is slowly improving, in particular for the Jupiter-family comets.
\end{abstract}

Keywords. Comets, orbit; impacts; Earth

\section{Introduction}

Comets pose a number of challenging problems when attempting to assess the impact hazard at the Earth. Long-period comets enter the planetary region from the Oort cloud (Weissman 1996, 1997) at random times and from random directions, making detection and prediction of potential impacts more difficult, and providing perilously short warning times. Determination of the sizes and masses of cometary nuclei is difficult, requiring specialized observations (Weissman \& Lowry 2003; Meech et al. 2004; Lamy et al. 2004), some of which are not possible without spacecraft flyby or rendezvous missions. Even those comets classified as periodic can have intervals of up to 200 years between perihelion passages, and thus the time to catalog all of the periodic comets with telescopic surveys can be quite long. The problem is also complicated by observational selection effects whereby many comets are missed if they pass perihelion (where they are brightest) on the opposite side of the Earth's orbit.

The number of Earth-crossing comets (ECCs) per year is considerably less than nearEarth asteroids (NEAs). However, the higher eccentricities and inclinations of cometary orbits as compared with NEAs lead to far higher encounter velocities with the Earth. Thus, cometary impactors are potentially far more energetic than asteroids for bodies of comparable mass.

Moreover, observed ECCs include comets that are far larger than any currently known NEA. For example, comet Hale-Bopp, a long-period comet that passed perihelion inside the Earth's orbit in 1997, had an estimated nucleus diameter of 27-42 km (Weaver

$\dagger$ Present address: Jet Propulsion Laboratory, 4800 Oak Grove Drive, Mail stop 183-301, Pasadena, CA 91109 USA 
et al. 1997). Taking a median value of $35 \mathrm{~km}$ and assuming a mean bulk density of $0.6 \mathrm{~g} \mathrm{~cm}^{-3}$ (Lowry \& Weissman 2003; Weissman et al. 2004) results in an estimated mass of $1.3 \times 10^{19} \mathrm{~g}$. The impact probability for Hale-Bopp on the Earth is $2.54 \times 10^{-9}$ per perihelion passage, fairly typical for a long-period comet. Because of the comet's high orbital eccentricity, 0.9951, and inclination, 89.43 degrees, the impact velocity would be $52.5 \mathrm{~km} \mathrm{sec}^{-1}$. The resulting impact energy is $1.9 \times 10^{32}$ ergs, equivalent to to $4.4 \times 10^{9}$ megatons. This is $\sim 44$ times the estimated energy of the Cretaceous-Tertiary extinction impactor $65 \mathrm{Myr}$ ago, the event that wiped out the dinosaurs. A Hale-Bopp like impact would probably be a planet sterilizing event.

This paper will review our current knowledge of the impactor flux at the Earth from active comets and the estimated frequency of impacts. Dormant comets are not included in the flux statistics presented here. Since dormant comets are difficult to differentiate from NEAs, they are generally included in the discovery and flux statistics for NEAs. A discussion on dormant comets is included later in this paper.

\section{A cometary primer}

Cometary orbits are classified by their orbital period and by their Tisserand parameter (Levison 1996). Long-period comets (LPCs) have orbital periods $>200$ years. The choice of 200 years is somewhat arbitrary and largely reflects the lack of good orbital solutions for comets in the past. Short-period comets have orbital periods $<200$ years, and are subdivided into two dynamical groups: Jupiter-family comets (JFCs) with periods $<20$ years, and Halley-type comets (HTCs) with periods $20<\mathrm{P}<200$ years.

More formally, the JFCs have Tisserand parameters relative to Jupiter $>2$ and the HTCs and LPCs have Tisserand parameters $<2$. The Tisserand parameter is a pseudoconstant of the motion in the restricted 3-body problem, Sun-Jupiter-comet, and was devised to recognize returning comets even after they might have been perturbed by a close approach with Jupiter. Because of its large mass, Jupiter is the major perturber of comets in the solar system, with Saturn a distant second.

Some dynamicists define a third class of short-period comets, Encke-type, with Tisserand parameters $>3$. These are short-period comets that have evolved to orbits totally interior to Jupiter's orbit. For purposes of discussion here, we will include the Encke-type comets with the JFCs.

Comets are transient objects in the planetary region. They come from two cometary "reservoirs" located at large distances from the Sun. The Oort cloud (Oort 1950) is a roughly spherical cloud of several times $10^{12}$ comets with semi-major axes between $\sim 3,000$ and 100,000 AU. Oort cloud comets fill the gravitational sphere of influence of the Sun out to $\sim 200,000$ AU, or approximately 1 parsec. Their orbits have been randomized by perturbations from the galactic tide and from random passing stars. These perturbations occasionally act to throw comets back into the planetary region where they can be observed (Dones et al. 2004).

It is generally agreed that Oort cloud comets are icy planetesimals thrown out of the giant planets region following the formation of the giant planets (Dones et al. 2004) Although the clearing of the planetary zones ejected most comets to interstellar space, a small fraction were captured to these very distant bound orbits. The Oort cloud is the source of the long-period comets. LPCs have random orbital inclinations and high eccentricities, approaching 1.

The second cometary reservoir is the Kuiper belt, located beyond the orbit of Neptune (Fernández 1980; Duncan et al. 1988). The Kuiper belt consists of two dynamically distinct populations: the "classical Kuiper belt" in low inclination, low eccentricity 
orbits beyond Neptune (plus some higher eccentricity and inclined objects captured into mean-motion resonances with Neptune), and the "scattered disk" in higher eccentricity, higher inclination orbits (though still relatively modest compared with the Oort cloud) that generally have perihelia near Neptune. Each of these sub-populations is estimated to contain $\sim 10^{9}$ comets $>1 \mathrm{~km}$ in radius.

The Kuiper belt is the source of the Jupiter-family comets. More specifically, because it is dyanmically interacting with Neptune, the scattered disk is considered the primary source for the JFCs (Duncan \& Levison 1997). JFCs have dynamically evolved from the scattered disk to orbits with perihelia in the terrestrial planets region. The Centaurs, objects in orbits between Jupiter and Neptune, are believed to be that transiting population. Most JFCs are Jupiter-crossing with orbits with moderate eccentricities, typically $\sim 0.6$, and moderate inclinations, $\sim 5-25$ degrees. Both of these are considerably higher than the corresponding values for NEA orbits.

The source of the Halley-type comets is still debated and could be the Oort cloud or the Kuiper belt, or some combination of the two (Levison et al. 2001). The HTC orbits have substantially higher eccentricities and inclinations than the JFCs, but are not yet fully randomized like the LPCs.

Cometary nuclei are typically $1-10 \mathrm{~km}$ in radius, irregularly shaped, and are composed of an icy-conglomerate mixture of ices (primarily water ice), organics and silicate dust, in roughly equal proportions. Bulk densities of cometary nuclei have been estimated to be between 0.3 and $1.0 \mathrm{~g} \mathrm{~cm}^{-3}$ (Weissman et al. 2004) with a most likely value of 0.6 $\mathrm{g} \mathrm{cm}^{-3}$. Like many asteroids, comets are believed to be weakly-bonded "rubble piles" of smaller icy planetesimals (Weissman 1986). Cometary nuclei are often observed to spontaneously split and shed one or many smaller pieces; random disruption is likely the principal physical loss mechanism for cometary nuclei (Weissman 1979).

Because of their lower densities and lower material strengths as compared with NEAs, cometary nuclei smaller than $\sim 300$ meters are not believed to survive atmospheric entry at the Earth, and likely explode relatively high in the atmosphere (Chyba et al. 1993). Thus, comets likely do not contribute significantly to craters less than a few kilometers in diameter on the Earth.

\section{Cometary impact rates and encounter velocities}

The impact probability, $p$, for an Earth-crossing comet, per perihelion passage, can be calculated using Öpik's classic equation (Öpik 1951)

$$
p=\frac{s^{2} U}{\pi \sin i\left|U_{x}\right|}
$$

where $s$ is the capture radius of the target planet (including gravitational focusing), $U$ is the encounter velocity of the comet with the Earth, $i$ is the inclination of the comet's orbit relative to the ecliptic, and $U_{x}$ is the component of the encounter velocity in the radial direction. The encounter velocity $U$ (in units of the Earth orbital velocity) is given by

$$
U=\sqrt{3-1 / a-2 \sqrt{a\left(1-e^{2}\right)} \cos i}
$$

where $a, e$, and $i$ are the semi-major axis, eccentricity, and inclination of the comet's orbit. The encounter velocity $U$ is also known as the hyperbolic excess velocity or the velocity at infinity, and represents the velocity of the comet relative to the Earth as it crosses the Earth's orbit, but not yet perturbed by the Earth's gravity. The impact velocity, $V_{i}$, the velocity at which the comet impacts the Earth's surface (not accounting 
for atmosphereic effects) is given by

$$
V_{i}=\left(U^{2}+V_{e}^{2}\right)^{1 / 2}
$$

where $V_{e}$ is the escape velocity from the Earth's surface, equal to $11.18 \mathrm{~km} \mathrm{sec}^{-1}$.

The Öpik equation has singularities for comets with perihelion distances close to $1 \mathrm{AU}$ and/or with inclinations close to 0 or 180 degrees. Also, the equation assumes a circular orbit for the Earth, whereas the Earth's orbital eccentricity is 0.0167. As a result, more detailed means of calculating the impact probability have been devised that eliminate these shortcomings (e.g. Kessler 1981). These more complex methods for finding the impact probability have been used in the estimates below.

Impact probabilities and velocities for long- and short-period comets have been calculated by a number of researchers: Weissman 1982; Weissman 1989; Marsden \& Steel 1994; Shoemaker et al. 1994. These papers are all in good general agreement among themselves and with the results presented herein.

\subsection{Long-period comets}

For long-period comets, a random sample of say $10^{7}$ hypothetical comets can be used to estimate the mean impact probability and encounter velocity, assuming a random distribution of orbital inclinations and a uniform perihelion distribution for the LPCs passing interior to $1 \mathrm{AU}$. The resulting mean impact probability is $2.2 \times 10^{-9}$ per comet per perihelion passage, with a mean encounter velocity of $50.5 \mathrm{~km} \mathrm{sec}^{-1}$. If one weights the individual encounter velocity estimates by the impact probability for each comet, the most probable encounter velocity is $53.5 \mathrm{~km} \mathrm{sec}^{-1}$. This results because high impact probabilities and high encounter velocities are correlated for retrograde comets, those in orbits going around the Sun in the opposite direction to the Earth's revolution.

Everhart (1967) corrected the observed orbital element distributions for LPCs for observational selection effects and found that the perihelion distribution was not uniform inside the Earth's orbit; it increased linearly from a relative value of 0.45 at $q \sim 0.01 \mathrm{AU}$ to 1.0 at $\mathrm{q}=1 \mathrm{AU}$. Using this perihelion distribution, the mean impact probability rises to $2.4 \times 10^{-9}$ per comet per perihelion passage. The mean and most probable encounter velocities remain almost identical with that for the uniform perihelion distribution at 50.5 and $53.6 \mathrm{~km} \mathrm{sec}^{-1}$.

\subsection{Jupiter-family comets}

There are 21 known Earth-crossing Jupiter-family comets, as of August 2006. Of these, 9 have been observed on more than one apparition, 8 are single apparition objects ( 7 of which were discovered by the automated telescopic surveys searching for NEOs), and 4 have been either destroyed or lost, or are no longer in an Earth-crossing orbit. Interestingly, the closest observed approach to the Earth by an active comet was comet D/1770 L1 Lexell in 1770. This comet was thrown into an Earth-crossing orbit by Jupiter in 1767, approached the Earth to $0.0146 \mathrm{AU}$ (about 2 million $\mathrm{km}$ ) in 1770, and after two orbits around the Sun again encountered Jupiter (with closest approach at $~ 3.5$ Jupiter radii) in 1779 and was removed from the terrestrial planets region.

The Earth-crossing JFCs are listed in Table 1. The mean impact probability is $8.4 \times$ $10^{-9}$ per perihelion passage or $1.6 \times 10^{-9}$ per year. The mean encounter velocity is $19.9 \mathrm{~km} \mathrm{sec}^{-1}$ and the most probable encounter velocity is $17.0 \mathrm{~km} \mathrm{sec}^{-1}$. The lower most probable encounter velocity results because of the relatively low inclination distribution of the JFCs, generally $<30$ degrees, which results in higher impact probabilities being correlated with lower encounter velocities. 
Table 1. Impact probabilities and encounter velocities for Earth-crossing JFCs

\begin{tabular}{|c|c|c|c|c|c|c|c|}
\hline Name & $\stackrel{q}{\mathrm{AU}}$ & e & $\begin{array}{c}\mathrm{i} \\
\mathrm{deg}\end{array}$ & $\begin{array}{l}\text { Period } \\
\text { yrs }\end{array}$ & $\begin{array}{l}\text { Impact } \mathrm{P} \\
\text { per orbit }\end{array}$ & $\begin{array}{l}\text { Impact } P \\
\text { per year }\end{array}$ & $\stackrel{\mathrm{U}}{\mathrm{km} / \mathrm{s}}$ \\
\hline \multicolumn{8}{|l|}{ Multiple apparitions } \\
\hline $2 \mathrm{P} /$ Encke & 0.339 & 0.847 & 11.76 & 3.30 & $3.34 \mathrm{e}-09$ & $1.01 \mathrm{e}-09$ & 29.5 \\
\hline 26P/Grigg-Skjellerup & 0.997 & 0.664 & 21.09 & 5.11 & $8.55 \mathrm{e}-09$ & $1.67 \mathrm{e}-09$ & 15.3 \\
\hline 45P/Honda-Mrkos-Pajdusakova & 0.530 & 0.825 & 4.25 & 5.27 & $9.32 \mathrm{e}-09$ & $1.77 \mathrm{e}-09$ & 24.7 \\
\hline 72P/Denning-Fukiwara & 0.780 & 0.820 & 8.64 & 9.02 & $5.58 \mathrm{e}-09$ & $6.18 \mathrm{e}-10$ & 18.8 \\
\hline 73P/Schwassmann-Wachmann 3 & 0.939 & 0.694 & 11.39 & 5.38 & 7.83e-09 & $1.46 \mathrm{e}-09$ & 13.1 \\
\hline 96P/Machholz 1 & 0.124 & 0.959 & 60.18 & 5.26 & $8.73 \mathrm{e}-10$ & $1.66 \mathrm{e}-10$ & 43.7 \\
\hline 141P/Machholz 2 & 0.753 & 0.750 & 12.80 & 5.23 & $3.81 \mathrm{e}-09$ & $7.29 \mathrm{e}-10$ & 19.2 \\
\hline 169P/NEAT & 0.605 & 0.768 & 11.32 & 4.21 & $3.76 \mathrm{e}-09$ & $8.92 \mathrm{e}-10$ & 22.5 \\
\hline D/1819 W1 Blanpain & 0.892 & 0.670 & 9.11 & 4.44 & $7.51 \mathrm{e}-09$ & $1.69 \mathrm{e}-09$ & 13.7 \\
\hline \multicolumn{8}{|l|}{ Single apparitions } \\
\hline P/2001 J1 NEAT & 0.937 & 0.758 & 10.16 & 7.62 & $8.28 \mathrm{e}-09$ & $1.09 \mathrm{e}-09$ & 13.6 \\
\hline P'/2001 Q2 Petriew & 0.946 & 0.696 & 13.94 & 5.49 & $6.89 \mathrm{e}-09$ & $1.26 \mathrm{e}-09$ & 13.8 \\
\hline P/2001 WF2 LONEOS & 0.976 & 0.667 & 16.92 & 5.02 & $9.68 \mathrm{e}-09$ & $1.93 \mathrm{e}-09$ & 13.8 \\
\hline P/2003 K2 Christensen & 0.549 & 0.829 & 10.14 & 5.75 & $3.99 \mathrm{e}-09$ & $6.93 \mathrm{e}-10$ & 24.9 \\
\hline P/2004 CB LINEAR & 0.912 & 0.689 & 19.15 & 5.02 & $4.17 \mathrm{e}-09$ & $8.30 \mathrm{e}-10$ & 16.5 \\
\hline P/2004 R1 McNaught & 0.988 & 0.682 & 4.89 & 5.48 & $3.35 \mathrm{e}-08$ & $6.12 \mathrm{e}-09$ & 10.0 \\
\hline P/2004 X1 LINEAR & 0.782 & 0.727 & 5.14 & 4.85 & $9.63 \mathrm{e}-09$ & $1.99 \mathrm{e}-09$ & 17.0 \\
\hline P/2005 JQ5 Catalina & 0.826 & 0.694 & 5.70 & 4.43 & $9.59 \mathrm{e}-09$ & $2.16 \mathrm{e}-09$ & 15.3 \\
\hline \multicolumn{8}{|l|}{$\begin{array}{l}\text { Destroyed, lost or no } \\
\text { longer Earth-crossing }\end{array}$} \\
\hline 3D/Biela & 0.879 & 0.751 & 13.22 & 6.63 & 4.83 & $7.27 \mathrm{e}-10$ & 16.1 \\
\hline $5 \mathrm{D} /$ Brorsen & 0.590 & 0.810 & 29.38 & 5.47 & $1.63 \mathrm{e}-09$ & $2.98 \mathrm{e}-10$ & 27.6 \\
\hline D/1766 G1 Helfenzrieder & 0.406 & 0.848 & 7.87 & 4.37 & $4.93 \mathrm{e}-09$ & $1.13 \mathrm{e}-09$ & 28.1 \\
\hline D/1770 L1 Lexell & 0.674 & 0.786 & 1.55 & 5.59 & $2.76 \mathrm{e}-08$ & $4.94 \mathrm{e}-09$ & 20.5 \\
\hline
\end{tabular}

\subsection{Halley-type comets}

There are 16 known Earth-crossing Halley-type comets, including 9 seen on more than one apparition, 6 seen on only one apparition (only 1 of which was discovered by the automated NEO surveys), and 1 that is lost. The prototype for this class, comet $1 \mathrm{P} / \mathrm{Halley}$, has been observed on every apparition since 240 B.C. and was last seen in 1986. It is a particularly large and active comet, with nucleus dimensions of $\sim 16 \times 8 \mathrm{~km}$. It was the first cometary nucleus encountered by flyby spacecraft.

The Halley-type comets are listed in Table 2 . The mean impact probability is $4.8 \times 10^{-9}$ per perihelion passage or $1.2 \times 10^{-10}$ per year. The much lower impact probability per year results from the considerably longer orbital periods of the HTCs. The mean encounter velocity is $46.0 \mathrm{~km} \mathrm{sec}^{-1}$ and the most probable encounter velocity is $51.3 \mathrm{~km} \mathrm{sec}^{-1}$. The higher most probable encounter velocity results because of the large number of retrograde HTCs with relatively high inclinations, which correlate with higher impact probabilities for those retrograde comets.

\section{Cometary fluxes and total impact rates}

There is considerable uncertainty as to the total flux of Earth-crossing comets. Everhart (1967) estimated that 11 LPCs brighter than $\mathrm{H}_{10}=11$ passed within $1 \mathrm{AU}$ of the Sun per year, yet only 2 or 3 of these were actually observed. Everhart showed that 
Table 2. Impact probabilities and encounter velocities for Earth-crossing HTCs

\begin{tabular}{|c|c|c|c|c|c|c|c|}
\hline Name & $\stackrel{q}{A U}$ & e & $\begin{array}{c}\mathrm{i} \\
\mathrm{deg}\end{array}$ & $\begin{array}{c}\text { Period } \\
\text { yrs }\end{array}$ & $\begin{array}{l}\text { Impact } \mathrm{P} \\
\text { per orbit }\end{array}$ & $\begin{array}{l}\text { Impact } P \\
\text { per year }\end{array}$ & $\begin{array}{c}\mathrm{U} \\
\mathrm{km} / \mathrm{s}\end{array}$ \\
\hline \multicolumn{8}{|l|}{ Multiple apparitions } \\
\hline 1P/Halley & 0.586 & 0.967 & 162.26 & 74.83 & $4.90 \mathrm{e}-09$ & $6.54 \mathrm{e}-11$ & 66.4 \\
\hline $8 \mathrm{P} /$ Tuttle & 0.997 & 0.824 & 54.71 & 13.48 & $5.36 \mathrm{e}-09$ & & 33.4 \\
\hline $12 \mathrm{P} /$ Pons-Brooks & 0.774 & 0.955 & 74.18 & 71.33 & $1.47 \mathrm{e}-09$ & $06 \mathrm{e}-11$ & 44.7 \\
\hline 23P/Brorsen-Metcalf & 0.479 & 0.972 & 19.33 & 70.76 & $2.06 \mathrm{e}-09$ & $1 \mathrm{e}-11$ & 31.0 \\
\hline $27 \mathrm{P} /$ Cror & 0.735 & 0.919 & 29.10 & 27.33 & 09 & 11 & 26.6 \\
\hline 35P/Herschel-Rigollet & 0.748 & 0.974 & 64.21 & 154.31 & $1.36 \mathrm{e}-09$ & $1 \mathrm{e}-12$ & 41.0 \\
\hline $55 \mathrm{P} / \mathrm{Ter}$ & 0.976 & 0.906 & 162.49 & 33.46 & $2.53 \mathrm{e}-08$ & -10 & 69.8 \\
\hline 109P/Sr & 0.960 & 0.963 & 113.45 & 132.16 & -09 & $3.72 \mathrm{e}-11$ & 59.9 \\
\hline $122 \mathrm{P} /$ de Vico & 0.659 & 0.963 & 85.38 & 75.17 & $1.25 \mathrm{e}-09$ & $1.67 \mathrm{e}-11$ & 49.3 \\
\hline \multicolumn{8}{|l|}{ Single apparition } \\
\hline $\mathrm{D} / 1917 \mathrm{I}$ & 0.190 & 0.993 & 32.68 & 141.41 & $1.26 \mathrm{e}-09$ & & 41.0 \\
\hline D/1937 D1 Wil & 0.619 & 0.981 & 26.02 & 185.95 & $1.72 \mathrm{e}-09$ & -12 & 29.2 \\
\hline D/1989 A3 Bradfie & 0.420 & 0.978 & 83.07 & 83.41 & 9.5 & e-11 & 49.0 \\
\hline P/1991 L3 Levy & 0.987 & 0.929 & 19.16 & 51.83 & $1.25 \mathrm{e}-08$ & $2.41 \mathrm{e}-10$ & 16.9 \\
\hline C/2001 OG108 & 0.994 & 0.925 & 80.25 & & & $9.05 \mathrm{e}-11$ & 46.4 \\
\hline $\mathrm{P} / 2005$ T4 SWAN & 0.649 & 0.930 & 160.04 & 28.23 & $4.87 \mathrm{e}-09$ & $1.72 \mathrm{e}-10$ & 66.4 \\
\hline \multicolumn{8}{|l|}{ Lost } \\
\hline D/1827 M1 Pons-C & 0.807 & 0.946 & 136.46 & 57.77 & $3.13 \mathrm{e}-09$ & $5.42 \mathrm{e}-11$ & 64.8 \\
\hline
\end{tabular}

observational selection effects led to many comets being missed, for example when they arrived at perihelion on the opposite side of the Sun from the Earth.

$\mathrm{H}_{10}$ is the magnitude of the active comet, with coma, as viewed from a distance of 1 AU from the Earth at zero phase, when the comet is 1 AU from the Sun (somewhat equivalent to the absolute magnitude for an asteroid). The estimate of $\mathrm{H}_{10}$ assumes that the cometary brightness varies as $r^{-4} d^{-2}$ where $r$ and $d$ are the heliocentric and geocentric distances, respectively. Although this provides an acceptable first-order estimate, brightening rates versus heliocentric distance vary considerably from one comet to the next. The rates appear to correlate with age, though age itself is often difficult to determine. Dynamically new LPCs exhibit brightening exponents as low as $r^{-2}$ on the inbound legs of their initial orbits, whereas JFCs often display very steep lightcurves, brightening as $r^{-6}$ or even higher. Unfortunately, there is no good conversion from $\mathrm{H}_{10}$ to nucleus dimensions, as the active fraction of the surfaces of cometary nuclei can also vary considerably from one comet to the next.

Levison (personal communication) estimates that there are $9 \times 10^{6}$ comets in the Oort cloud with perihelia $\leqslant 1$ AU. Given a typical orbital period of 4 Myr for an LPC entering the planetary system for the first time from the Oort cloud, this translates into 2.2 dynamically new LPCs per year. Since the average LPC makes 5 passages through the planetary system (Weissman 1979), the total flux of LPCs is $~ 11$ ECC per year, in surprisingly good agreement with the Everhart estimate.

Discovery statistics for LPCs show a steady increase in the rate of discoveries over time. Since 1900 there have been 414 discoveries of LPCs with perihelia $<3$ AU, not including Sun-grazing comets with $\mathrm{q}<0.08$ AU. The first 207 of these were found from 1900-1978, an interval of 79 years, while the next 207 were found from 1979-2006, an interval of only 27.6 years. Further dividing the second interval, one finds that 105 LPCs were found from 1979-1997 (19 years) versus 102 LPCs found from 1998-2006 (8.6 years). 
The increase in discovery rate is clearly associated with the automated telescopic surveys searching for NEAs. From 1900-1978 the discovery rate of long-period ECCs was 2.6 per year. From 1979-1997 it was 2.3 long-period ECCs per year, statistically the same. But from 1998-2006 the rate increased to 4.3 long-period ECCs per year. Although this rate is still short of the Everhart and Levison estimates, it is conceivable that the more sensitive surveys planned for future years may eventually yield rates comparable to those values. Discoveries of JFCs and HTCs have also been aided by the automated surveys.

Given a rate of 11 long-period ECCs per year, the total impact rate is $2.6 \times 10^{-8}$ per year, or one impact every $38 \mathrm{Myr}$. Comet showers caused by random stars passing directly through the Oort cloud will double the mean impact rate over time, though the showers come in bursts lasting only 2-3 Myr. We are currently not in a cometary shower.

Unfortunately, it is not currently possible to estimate the sizes of the nuclei associated with this flux. There exist very few reliable estimates of the radii of LPCs. Even the estimate for the nucleus of comet Hale-Bopp in Section 1 is highly uncertain.

Estimates of the total flux of Earth-crossing JFCs are also somewhat indefinite. New Earth-crossing JFCs continue to be discovered by the automated NEO surveys and by amateur observers. As a rough guess, we might assume that the current discovery of Earth-crossing active JFCs is $50 \%$ complete. Then the average impact rate is $6.7 \times 10^{-8}$ per year, or one impact every $15 \mathrm{Myr}$.

A similar calculation can be made for the HTCs. Discovery completeness for this population is lower because of their longer orbital periods. If we assume that completeness is $33 \%$, then the impact rate is $5.8 \times 10^{-9}$ per year or one impact every $176 \mathrm{Myr}$. If we assume that completeness is only $10 \%$, then the average impact rate is $1.9 \times 10^{-8}$ per year or one impact every 52 Myr.

Again, the problem is matching this impactor flux with the sizes of the impactors and predicting the energy of the impacts. Progress has been made in estimating the sizes of JFCs and HTCs through a variety of observational techniques. Because these comets are periodic, their returns can be predicted very accurately, and they can be observed at distances of 4-5 AU from the Sun on the inbound legs of their orbits, where they are most likely to be inactive. Brightness estimates can then be converted to nucleus radius estimates by assuming a typical cometary albedo of 0.04 . Another successful method for estimating the sizes of cometary nuclei is to use the high angular resolution of the Hubble Space Telescope to image active comets when they are close to the Earth, and then model and subtract the coma signal from the total brightness. Again, a typical cometary albedo of 0.04 needs to be assumed. Lastly, four cometary nuclei (Halley, Borrelly, Wild 2, and Tempel 1) have been directly imaged by spacecraft flybys. Over 65 JFCs and HTCs have had their radii estimated in this way (Weissman \& Lowry 2003, Meech et al. 2004, Lamy et al. 2004).

This include six of the JFCs listed in Table 1, whose radius estimates range from 0.17 to $4.4 \mathrm{~km}$, the smallest being comet Blanpain and the largest comet Encke. In many cases, radius estimates by different observers agree well, but in other cases, such as Encke, there is considerable disagreement on the size of the nucleus. The radius estimates for JFCs (and HTCs) are shown in Table 3.

Radius estimates also exist for four of the HTCs in Table 2. These range from 1.78 to $15.0 \mathrm{~km}$; the smallest corresponds to comet Tempel-Tuttle and the largest to comet Swift-Tuttle. Size estimates for HTCs are typically much larger than for JFCs. However, these four Earth-crossing HTCs are the only HTCs for which reliable radius estimates exist, versus $\sim 60$ measured JFCs. There are strong observational biases here, as there are far fewer opportunities to measure the radii of HTCs because of their longer orbital 
Table 3. Nucleus radius estimates for periodic ECCs

\begin{tabular}{lccl}
\hline Name & $\begin{array}{c}\text { Impact } \mathrm{P} \\
\text { per year }\end{array}$ & $\begin{array}{c}\mathrm{U} \\
\mathrm{km} / \mathrm{s}\end{array}$ & $\begin{array}{l}\text { Radius estimates } \\
\mathrm{km}\end{array}$ \\
\hline Jupiter-family comets & & & \\
2P/Encke & $1.01 \mathrm{e}-09$ & 29.5 & $3.4,2.4,4.0,4.4$ \\
26P/Grigg-Skjellerup & $1.67 \mathrm{e}-09$ & 15.3 & $1.4,1.44,2.90$ \\
45P/H-M-P & $1.77 \mathrm{e}-09$ & 24.7 & $0.34,1.34$ \\
73P/S-W 3 & $1.46 \mathrm{e}-09$ & 13.1 & $0.6,0.4$ \\
96P/Machholz 1 & $1.66 \mathrm{e}-10$ & 43.7 & 3.3 \\
D/1819 W1 Blanpain & $1.69 \mathrm{e}-09$ & 13.7 & 0.17 \\
& & & \\
Halley-type comets & & & \\
1P/Halley & $6.54 \mathrm{e}-11$ & 66.4 & $5.73,5.25$ \\
8P/Tuttle & $3.98 \mathrm{e}-10$ & 33.4 & 7.3 \\
55P/Tempel-Tuttle & $7.55 \mathrm{e}-10$ & 69.8 & $1.78,1.80,1.85$ \\
109P/Swift-Tuttle & $3.72 \mathrm{e}-11$ & 59.9 & $11.8,13.73,15.0$ \\
\hline
\end{tabular}

periods. Also, the various techniques for estimating radii are most successful for nuclei that are relatively large and/or relatively inactive.

If one combines the impact rate estimates for LPCs, JFCs, and HTCs above, then the total impact rate is $1.1 \times 10^{-7}$ per year, or one impact every $9.1 \mathrm{Myr}$. If comet showers are included, these numbers increase to $1.4 \times 10^{-7}$ per year, or one impact every 7.2 Myr. These are only a few per cent of the rates for NEAs. However, as noted earlier, the higher encounter velocities and larger nuclei result in comets contributing significantly to the more energetic, though rare, impacts on the Earth.

\section{Dormant comets}

The most common loss mechanism for cometary nuclei is dynamical ejection from the solar system. Random disruption, or splitting, is likely the most common physical loss mechanism, whereby the weakly bonded nucleus fragments separate and rapidly sublimate away. A less common but possible mechanism is that the cometary nuclei evolve to a dormant state, in which a lag deposit of non-volatile material remains on the nucleus surface and slowly cuts off cometary activity. In the case of comet Halley, $\sim 70-80 \%$ of the sunlit surface appeared to be inactive. For most JFCs, the fraction of active surface area is typically only a few per cent.

In recent years there has been increasing evidence for dormant cometary nuclei among the NEA population. Bottke et al. (2002) showed that the orbital distribution of NEAs could best be explained if $\sim 6 \%$ of the NEA population had evolved from Jupiterfamily comet orbits. Weissman et al. (2002) examined the list of NEAs identified by Bottke et al. as likely dormant comets, and showed that a significant fraction were either low albedo or of a primitive taxonomic type, both consistent with a cometary origin. Fernández et al. (2005) showed that two-thirds of NEAs with Tisserand parameters $<3$, suggestive of a cometary origin, had low albedos, less than 0.075 . Some estimates have placed the fraction of dormant comets among the NEAs as high as $15 \%$.

An unusual object, 1996 PW, was discovered in 1996. This asteroidal-appearing object was in a highly eccentric orbit, similar to that of a long-period comet, with an orbital period of $\sim 4,000$ years. Weissman \& Levison (1997) showed that this object was equally likely to be a dormant long-period comet or an asteroid that had been ejected to the Oort cloud and was now returning. Color measurements suggested that 1996 PW was 
a D-type asteroid, consistent with an outer main belt asteroid or a primitive nucleus surface. No evidence of cometary activity was detected.

The actual fraction of comets that evolve to a dormant state is highly uncertain. The process is often described as "cometary fading," suggesting that active nuclei slowly become less luminous and eventually incapable of producing any visible coma. Cometary fading is often invoked by dynamical modelers as a convenient mechanism to account for the difference between predicted and observed numbers of comets in a particular dynamical class (e.g., Bailey 1984; Emel'yanenko \& Bailey 1996). However, the differences may also be the result of incorrect initial assumptions and/or poor modeling of other physical loss mechanisms or of the dynamics itself, as well as observational selection effects and incompleteness in the observed sample of comets and asteroids. Indeed, Levison et al. (2002) argued that cometary fading had to be a relatively minor end-state because of the relatively low numbers of dormant objects discovered in orbits that could have evolved from long-period comet orbits. Further studies of the fraction of dormant comets among the NEAs will be quite valuable in providing an improved understanding of "cometary fading" and its role in other cometary evolution problems.

\section{Summary and discussion}

Comets make up a small but important fraction of near-Earth objects. Although Earthcrossing comets are far less numerous than NEAs, their high orbital inclinations and eccentricities result in very high hyperbolic encounter velocities with the Earth, up to $72 \mathrm{~km} \mathrm{sec}^{-1}$. Additionally, some Earth-crossing comets are considerably larger than any known NEA. Thus, comets likely provide the largest and most energetic impactors on the Earth.

The total cometary impact rate on the Earth, based on observed comets and crudely correcting for observational incompleteness, is $1.4 \times 10^{-7}$ per year, or one impact every 7.2 Myr. About $48 \%$ of this total is due to Jupiter-family comets, $14 \%$ due to Halleytype comets, and $38 \%$ due to long-period comets, though half of the LPCs arrive in brief cometary showers of 2-3 Myr duration. We are currently not in a cometary shower. Most probable encounter velocities range between $17.0 \mathrm{~km} \mathrm{sec}^{-1}$ for JFCs, $51.3 \mathrm{~km} \mathrm{sec}{ }^{-1}$ for HTCs, and $53.6 \mathrm{~km} \mathrm{sec}^{-1}$ for LPCs.

It is difficult to compare these cometary impact rates with those for NEAs because the sizes of the cometary nuclei are not well known. However, progress is being made, particularly in the case of the JFCs. This is fortunate since JFCs appear to provide about half of the cometary impactors on the Earth. Unfortunately, the situation is considerably poorer in the case of the HTCs and LPCs. In addition, the long orbital periods of the HTCs mean that it will take at least several hundred years to survey this population, though technology improvements over that time may be able to speed the process. Lastly, the problem of the short warning time for LPCs on potential Earth-impacting orbits needs to be dealt with. LPCs are typically discovered at distances $<5$ AU from the Sun, $\leqslant 1$ year prior to perihelion passage. There are exceptions, such as comet Hale-Bopp, discovered at $7.14 \mathrm{AU}$, but still $<2$ years from perihelion. The next generation surveys are likely to improve the warning time but it is also likely that new technologies are required to make truly significant improvements.

The automated telescopic surveys for NEOs have been very successful at increasing our knowledge of the populations of comets in Earth-crossing orbits. This can be expected to improve further with the next generation of surveys, including PAN-STARRS and LSST. 


\section{Acknowledgements}

I thank Hans Rickman for useful comments on an earlier draft of this paper. This work was supported by the NASA Planetary Astronomy and Planetary Geology \& Geophysics Programs, and was performed at the Jet Propulsion Laboratory under a contract with NASA.

\section{References}

Bailey, M.E. 1984, MNRAS 211, 347

Bottke, W.F., Morbidelli, A., Jedicke, R., et al. 2002, Icarus 156, 399

Chyba, C.F., Thomas, P.J. \& Zahnle, K.J. 1993, Nature 361, 40

Dones, L., Weissman, P.R., Levison, H.F. \& Duncan, M.J. 2004, in: M.C. Festou, H.U. Keller \& H.A. Weaver (eds.), Comets II (University of Arizona Press, Tucson), p. 153

Duncan, M.J., Quinn, T. \& Tremaine, S. 1988, Astrophys. J. 328, 69

Duncan, M.J. \& Levison, H.F. 1997, Science 276, 1670

Emel'Yanenko, V.V. \& Bailey, M.E. 1996, Earth, Moon 63 Planets 72, 35

Everhart, E. 1967, Astron. J., 72, 1002

Fernández, J.A. 1980, MNRAS 192, 481

Fernández, Y.R., Jewitt, D.C. \& Sheppard, S.S. 2005, Astron. J. 130, 308

Kessler, D.J. 1981, Icarus 48, 39

Lamy, P.L., Toth, I., Fernández, Y.R. \& Weaver, H.A. 2004, in: M.C. Festou, H.U. Keller \& H.A. Weaver (eds.), Comets II (University of Arizona Press, Tucson), p. 223

Levison, H.F. 1996, ASP Conf. Ser. Completing the Inventory of the Solar System, 107, p. 173 Levison, H.F., Dones, L. \& Duncan, M.J. 2001, Astron. J. 121, 2253

Levison, H.F., Morbidelli, A., Dones, L., et al. 2002, Science 296, 2212

Lowry, S.C. \& Weissman, P.R. 2003, Icarus 164, 492

Marsden, B.G., \& Steel, D.I. 1994, in: T. Gehrels, M.S. Matthews \& A.M. Schumann (eds.), Hazards Due to Comets and Asteroids (University of Arizona Press, Tucson), p. 221

Meech, K.J., Hainaut, O.R. \& Marsden, B.G. 2004, Icarus 170, 463

Oort, J.H. 1950, Bull. Astron. Inst. Neth. 11, 91

Öpik, E.J. 1951, Proc. R. Irish Acad. 54, 165

Shoemaker, E.M., Weissman, P.R. \& Shoemaker, C.S. 1994, in: T. Gehrels, M.S. Matthews \& A.M. Schumann (eds.), Hazards Due to Comets and Asteroids (University of Arizona Press, Tucson), p. 313

Weaver, H.A., Feldman, P.D., A'Hearn, M.F. \& Arpigny, C. 1997, Science 275, 1900

Weissman, P.R. 1979, In Dynamics of the Solar System, p. 277

Weissman, P.R. 1982, In Geol. Soc. Amer. Special Paper 190: Geological Implications of Impacts of Large Asteroids and Comets on the Earth, p. 15

Weissman, P.R. 1989, In Geol. Soc. Amer. Special Paper 24\%: Global Catastrophes in Earth History, p. 211

Weissman, P.R. 1986, Nature 320, 242

Weissman, P.R. 1996, ASP Conf. Ser.: Completing the Inventory of the Solar System 107, p .265

Weissman, P.R. 1997, In Near-Earth Objects, Annals NY Acad. Sci. 822, 67

Weissman, P.R. \& Levison, H.F. 1997, Astrophys. J. Lett. 488133

Weissman, P.R., Bottke, W.F. \& Levison, H.F., 2002, in: W.F. Bottke, A. Cellino, P. Paolicchi \& R.P Binzel (eds.), Asteroids III (University of Arizona Press, Tucson), p. 669

Weissman, P.R. \& Lowry, S.C. 2003, LPSC 34, \#2003

Weissman, P.R., Asphaug, E. \& Lowry, S.C. 2004, in: M.C. Festou, H.U. Keller \& H.A. Weaver (eds.), Comets II (University of Arizona Press, Tucson), p. 337 